\title{
Microbial community structure in a biogas digester utilizing the marine energy crop Saccharina latissima
}

\author{
Phillip B. Pope $\cdot$ Vivekanand Vivekanand \\ Vincent G. H. Eijsink $\cdot$ Svein J. Horn
}

Received: 26 September 2012/ Accepted: 5 October 2012/Published online: 25 October 2012

(C) The Author(s) 2012. This article is published with open access at Springerlink.com

\begin{abstract}
Seaweed is a highly attractive marine crop for the production of biofuels, due to its rapid growth rate as well as high polysaccharide and low lignin content. One appealing exploitation route is the production of biogas by anaerobic digestion. Interestingly, despite the compositional differences between seaweed and lignocellulosic biomass, available data indicate that conditions and inocula traditionally used for the latter may work well for seaweed. To gain more insight into the underlying microbial processes, we have generated 16S rRNA gene amplicon pyrosequencing data to comparatively describe microbial communities in biogas digesters containing either the seaweed Saccharina latissima or wheat straw. The seaweed digesters gave better biogas yield and a higher relative abundance of core group Methanosaeta-affiliated Archaea. Conversely, variation in biomass had only minor abundance effects towards dominant bacterial lineages and influenced only low-abundant bacterial OTUs. Affiliations between dominant archaeal and bacterial phylotypes described here and previously identified anaerobic digestion core groups indicate that trends are beginning to emerge within these newly explored microbial ecosystems, the understanding of which is currently impeded by limited published datasets.
\end{abstract}

Keywords Biogas - Anaerobic digestion - Seaweed . Macroalgae $\cdot$ Methane

P. B. Pope $(\bowtie)$ · V. Vivekanand · V. G. H. Eijsink · S. J. Horn Department of Chemistry, Biotechnology and Food Science, Norwegian University of Life Sciences, Post Office Box 5003, 1432 Ås, Norway e-mail: phil.pope@umb.no
Biogas production, particularly in the purified form of biomethane, is seen as a vital component of renewable energy technologies due to the wide variety of organic sources that can be used and the compatibility of methane with existing energy infrastructure. Efforts to augment the biogas processes have focused on utilizing waste materials as well as alternative biomass substrates that lessen the impact on arable land. To that end, seaweed species have been identified as high potential substrates for biomethane production, due to their rapid growth rate as well as high polysaccharide $(\sim 60 \%)$ and low lignin content (Horn 2009). Compared to organic waste streams and terrestrial biomasses, relative little is known on the anaerobic digestion (AD) of marine substrates. Available data for seaweed are, however, quite promising, in particular for the brown seaweed Saccharina latissima (Nielsen and Heiske 2011; Vivekanand et al. 2012). Seaweed is less recalcitrant than lignocellulosic materials meaning that thermal pretreatments that are often used to speed up biogas processes can be milder, thus reducing the risk of inhibitor formation that is common during the harsher pretreatments (Vivekanand et al. 2012).

In this study, we report compositional and comparative analysis of the microbial communities in anaerobic digesters. 16S rRNA gene amplification for both bacterial and archaeal domains was performed to ensure that representatives for all the key metabolic stages of $\mathrm{AD}$ were enveloped, that is, polymer hydrolysis, sugar fermentation, acetogenesis (all Bacteria) and methanogenesis (Archaea). Samples were collected from three $1.1 \mathrm{~L}$ batch digesters run in triplicate for 119 days at $37^{\circ} \mathrm{C}$ (stable pH 7.3 over the course of the experiment). All three digesters were inoculated with $600 \mathrm{~mL}$ of pre-incubated waste water sludge containing $10.5 \mathrm{~g} \mathrm{~L}^{-1}$ of volatile solids (VS) (Vivekanand et al. 2012), and were defined according to 
the new substrate added (1.05 g VS added at day 0 and 67 , $2.1 \mathrm{~g}$ VS total): inoculum containing no additional organic substrate (IC), inoculum with seaweed ( $S$. latissima, $\mathrm{IC}+\mathrm{SW}$ ) and inoculum with steam exploded wheat straw (Triticum aestivum, IC $+\mathrm{WS}$ ). The total liquid volume in all digesters was then adjusted to $700 \mathrm{~mL}$ by adding distilled water. Total methane production in IC $+\mathrm{SW}$ $\left(223 \pm 61 \mathrm{~mL} \mathrm{~g}^{-1} \mathrm{VS}\right)$ was approximately twice as high as in IC + WS $\left(98 \pm 44 \mathrm{~mL} \mathrm{~g}^{-1} \mathrm{VS}\right)$; note that $\mathrm{SW}$ and WS materials have different $\mathrm{C} / \mathrm{N}$ ratios of 8.8 and 98.4 , respectively (Vivekanand et al. 2012). For each digester, sub-samples from each triplicate (equal volume) were pooled, and DNA extraction was performed as described by Rosewarne et al. (2010). Rrs genes were amplified using the broadly conserved primer sets 27F-515R [Bacteria: (Pope et al. 2012)] and 340F-1000R [Archaea: (Gantner et al. 2011)], both containing the 454 Life Sciences primer A sequence and a unique 8-nt multiplex identifier (Hamady et al. 2008). Rrs gene sequences were quality filtered using the QIIME software package (Caporaso et al. 2010), whilst error correction and chimera removal were performed using OTUPIPE which incorporates UCHIME (Edgar et al. 2011). Operational taxonomic units (OTUs) were clustered at $97 \%$ sequence identity using UCLUST software (Edgar 2010) and taxonomy was assigned using the Ribosomal Database Project classifier (Cole et al. 2003). After filtering and normalization (datasets randomly "subsampled" to remove sample heterogeneity), 1,992 bacterial and 651 archaeal 16S rRNA sequences (in total) clustered into 63 and 14 OTUs, respectively (Table 1; Acc. Numbers JX279942-JX280018). Rarefaction analysis showed that the three digester datasets afforded a similar degree of adequate coverage of bacterial biodiversity within each digester (Fig. 1; Table 1). Moreover, Fig. 1 illustrated that the addition of seaweed appears to reduce archaeal species diversity.

Comparisons of the archaeal communities revealed that OTUs ARC_nor-1, ARC_nor-2 and ARC_nor-3, affiliated to the Taxonomic Order-ranks Methanosarcinales, Methanomicrobiales and Methanobacteriales, respectively, were dominant in all three samples (Fig. 2a-b). However, their composition varied considerably depending on the digester substrate (Fig. 2b-c). The increased dominance of ARC_nor-1 in the IC + SW digester coincided with higher methane production [Fig. 2; (Vivekanand et al. 2012)], as well as a slightly higher methane content in the biogas (57\% vs. $53 \%$ in IC + WS). Affiliation of ARC_nor-1 to an acetoclastic methanogen (Methanosaeta concilii; $98 \%$ ID) was also in agreement with Methanosaeta dominance in $\mathrm{AD}$ communities that utilize freshwater algae substrates (Ellis et al. 2012). In contrast, hydrogenotrophic methanogens, of which ARC_nor-2 is putatively categorized, were most dominant in the inoculum digester (IC), and their relative abundance decreased in digesters containing either IC + WS or IC + SW (Fig. 2b). Interestingly, both ARC_nor-1 and ARC_nor-3 were affiliated (99\% ID; Table 1) to previously described and repeatedly detected core group phylotypes (OTU-VI and OTU-V, respectively), which dominate sludge $\mathrm{AD}$ communities (Rivière et al. 2009).

Spirochaetes, Bacteroidetes and Chloroflexi were the dominant bacterial phyla in all three samples (Fig. 3a). Dominance of these phyla, with the exception of the Spirochaetes, is commonly observed in biogas processes (Nelson et al. 2011), whilst the low relative abundance of Proteobacteria- and Firmicutes-affiliated OTUs is in contrast with previous studies that have demonstrated their abundance in AD reactors (Kampmann et al. 2012; Nelson et al. 2011). The majority of the bacterial OTUs were distantly related to cultured relatives, whereas close similarities were observed with previous biogas microbial community studies describing uncultured phylotypes (Table 1). In particular, both BAC_nor-3 and BAC_nor-4 exhibited $99 \%$ sequence identity to dominant Chloroflexiaffiliated OTUs that have been previously defined as highly prevalent core phylotypes involved in AD of sludge [Core group $\alpha$-III and $\alpha$-VI; (Rivière et al. 2009)]. The repeated detection of Chloroflexi-affiliated phylotypes in high abundance within biogas processes points towards a significant role and reveals a need for future investigations. Several OTUs of lower abundance demonstrated marked shifts depending on which substrate was present (Fig. 3bc). BAC_nor-13, a Petrobacter-affiliated betaproteobacteria decreased approximately seven-fold in IC $+\mathrm{SW}$ digesters, whereas, the Bacteroidales-affiliated BAC_nor12 and Victivallis-affiliated BAC_nor-26 experienced an eight-fold and nine-fold increase, respectively. The phenotype of BAC_nor-26 may be potentially interesting, as Victivallis sp. isolates have previously been described as capable of fermenting a variety of sugars including glucose and mannitol [found in brown seaweed; (Horn and Ostgaard 2001)] subsequently producing acetate, $\mathrm{H}_{2}$ and ethanol as end-products (Zoetendal et al. 2003).

The present study shows that the microbial consortia involved in $\mathrm{AD}$ of seaweed comprise deeply branched OTUs. However, there are indications that trends in $\mathrm{AD}$ microbial profiles are beginning to emerge with the detection of several previously identified core group archaeal and bacterial phylotypes (Table 1; Rivière et al. 2009). Compared to the IC + WS digester, the IC + SW digester showed some conspicuous differences, the most prominent being an increase in methane production and the relative abundance of the Methanosaeta concilii-affiliated (presumably acetoclastic) ARC_nor-1. Given that methanogens are believed to rely on syntrophic relationships with bacteria for key metabolites (i.e., acetate, $\mathrm{H}_{2} / \mathrm{CO}_{2}$ ), 


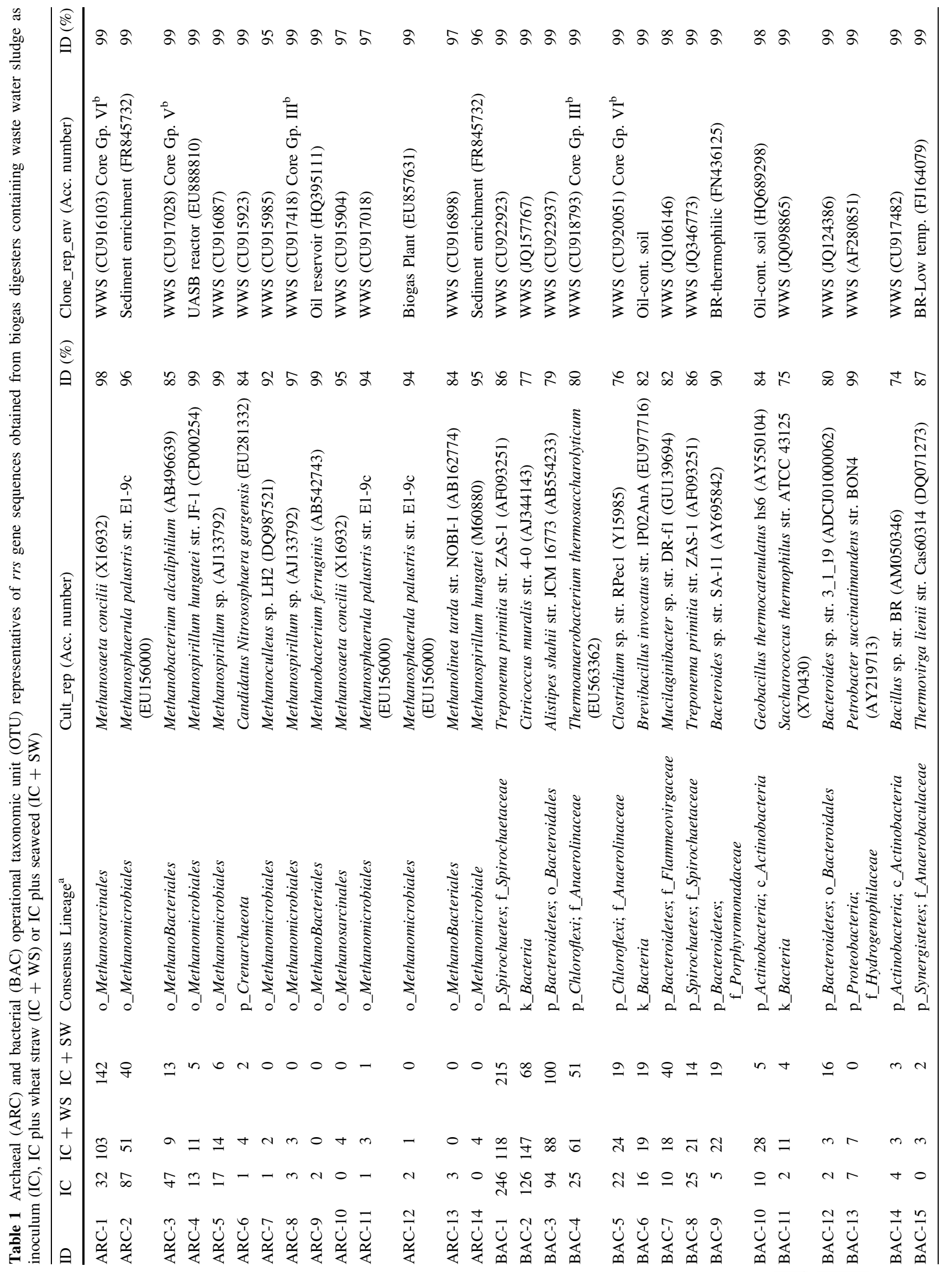









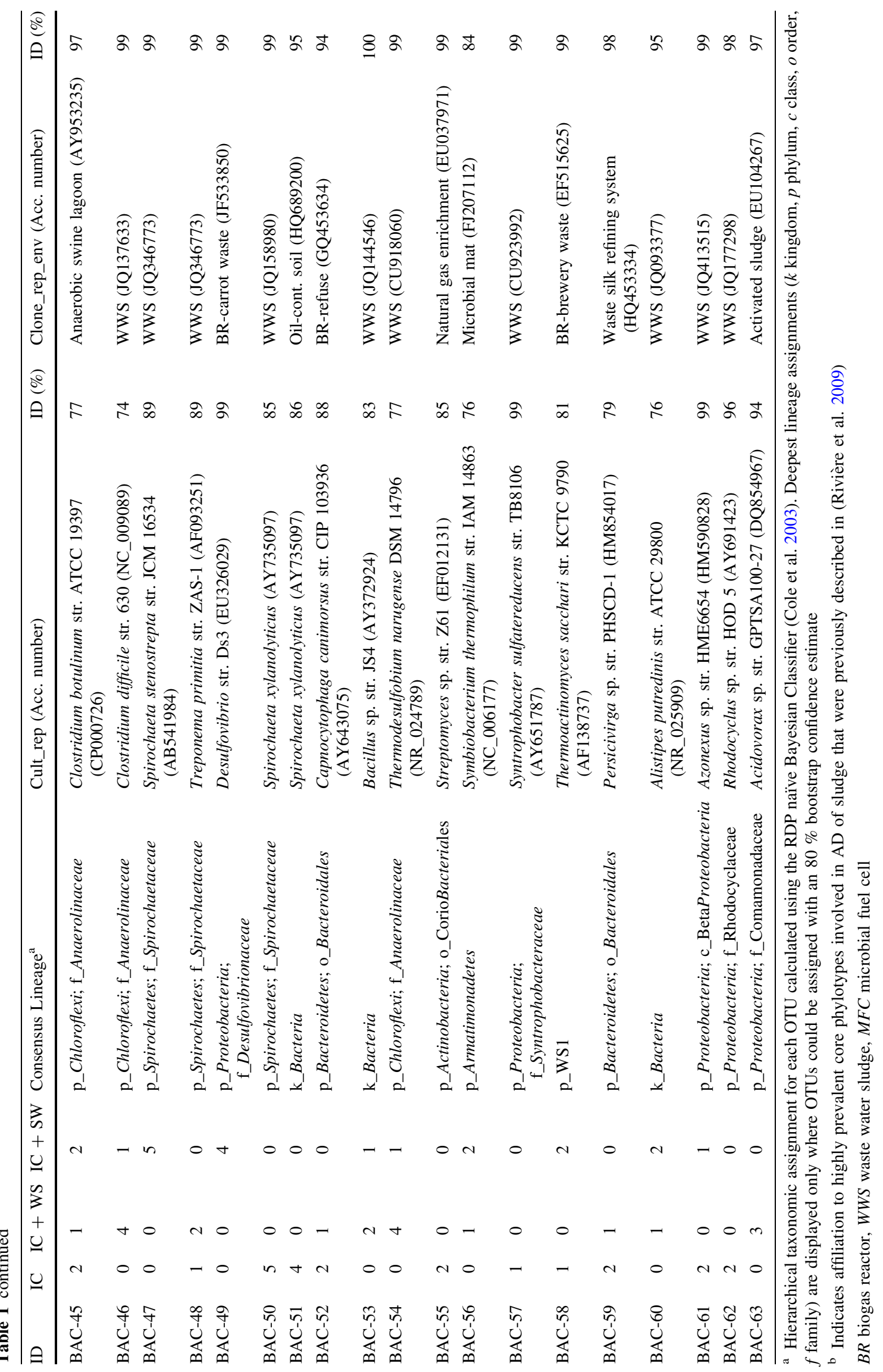




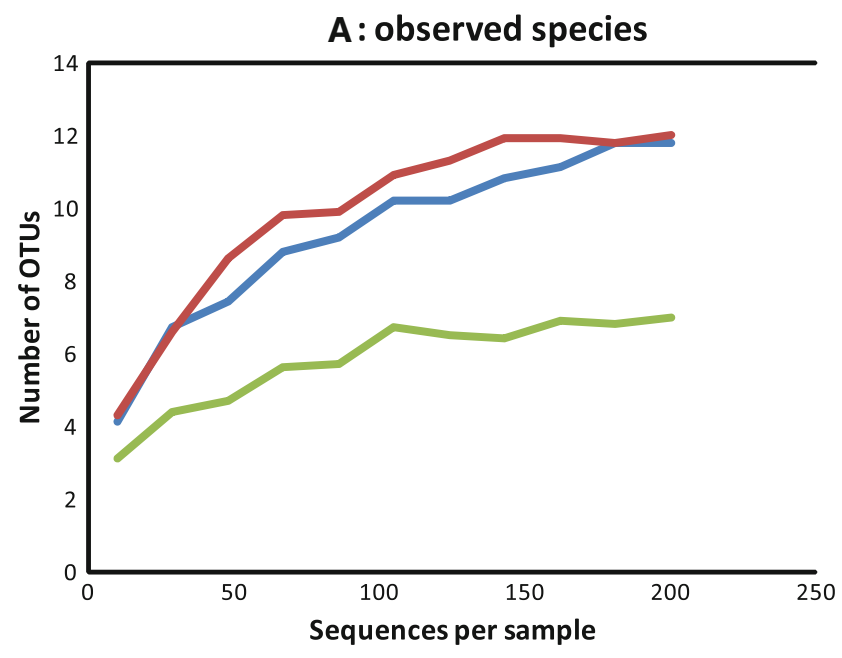

Fig. 1 Rarefaction analyses using operational taxonomic unit (OTU) frequency of archaeal (a) and bacterial (b) rrs gene datasets obtained from the biogas digesters containing waste water sludge as inoculum

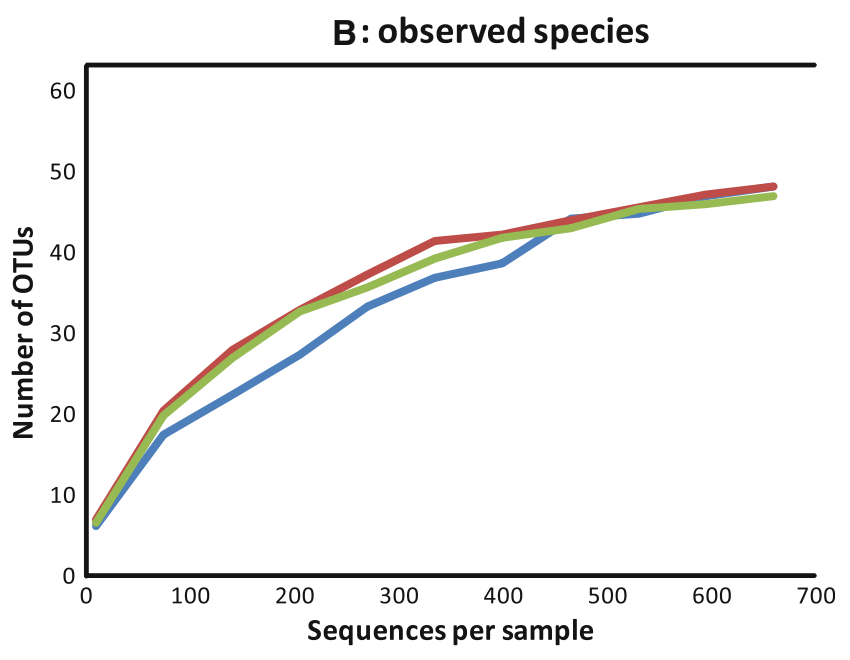

(IC blue), IC plus wheat straw (IC + WS red) or IC plus seaweed $(\mathrm{IC}+\mathrm{SW}$ green $)$. A $97 \%$ sequence identity threshold has been employed for the OTU constructions used in these analyses

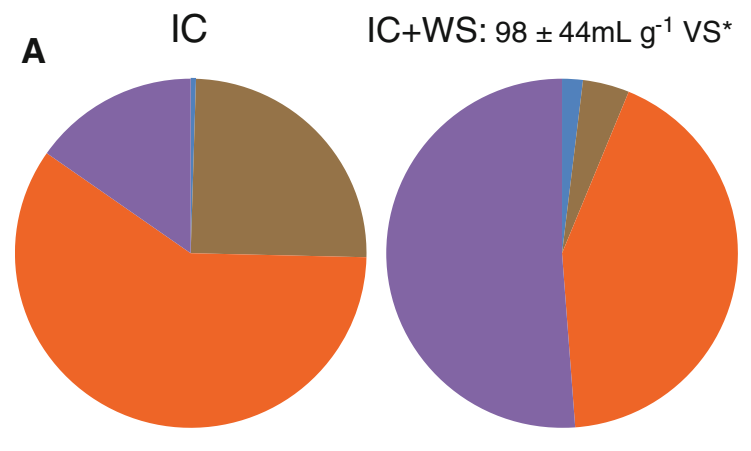

B

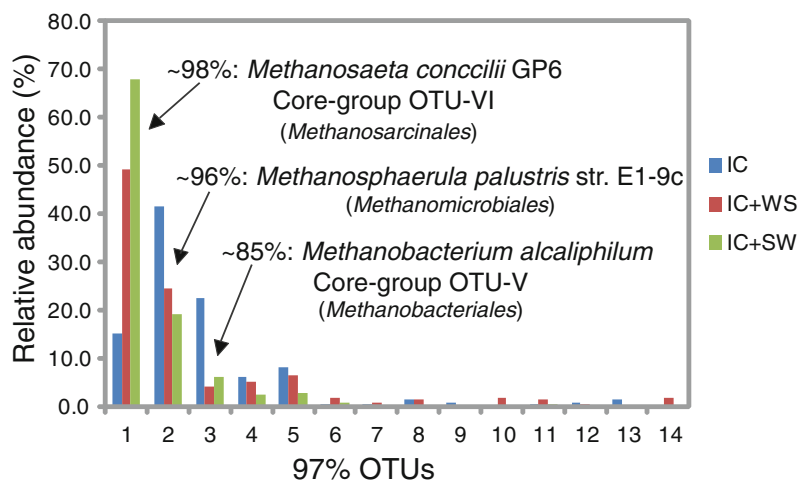

Fig. 2 Relative abundance and comparison profiles of archaeal 16S rRNA OTUs identified in anaerobic digesters containing either waste water sludge with no additional organic substrate (inoculum, IC), IC plus wheat straw (IC + WS), or IC plus seaweed $(\mathrm{IC}+\mathrm{SW})$. a, b The relative abundance of archaeal lineages at a phylum-level and OTU-level, respectively. OTU abundance shifts between WS and SW digesters $\mathbf{c}$ were measured as either fold-change increases $(+)$ or decreases (-) against IC measurements. Colour coding in $\mathbf{b}$ and $\mathbf{c}$ are as follows: blue indicates IC, red indicates IC $+\mathrm{WS}$ and green
IC+SW: $223 \pm 61 \mathrm{~mL} \mathrm{~g}^{-1} \mathrm{VS}^{*}$

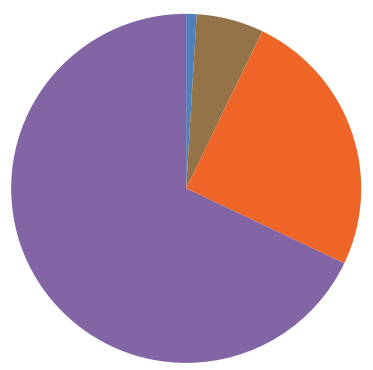

Crenarchaeota; C2; GrfC26

Euryarchaeota; Methanobacteria; Methanobacteriales Euryarchaeota; Methanomicrobia; Methanomicrobiales Euryarchaeota; Methanomicrobia; Methanosarcinales
C

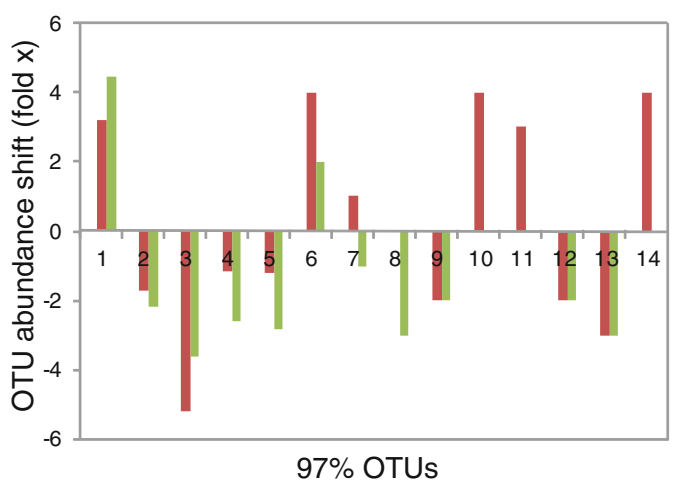

indicates IC + SW. Lineage information for selected OTUs and OTU affiliation to previously described, highly prevalent core phylotypes (Rivière et al. 2009) is provided. OTUs numbers in the $x$-axis correspond to ARC_nor-terminology referred to in the text. Total methane yields are included in a for IC + WS and IC + SW, which are provided in the original publication on methane production (Vivekanand et al. 2012) and normalized for production in IC. $V S^{*}$ volatile solids 

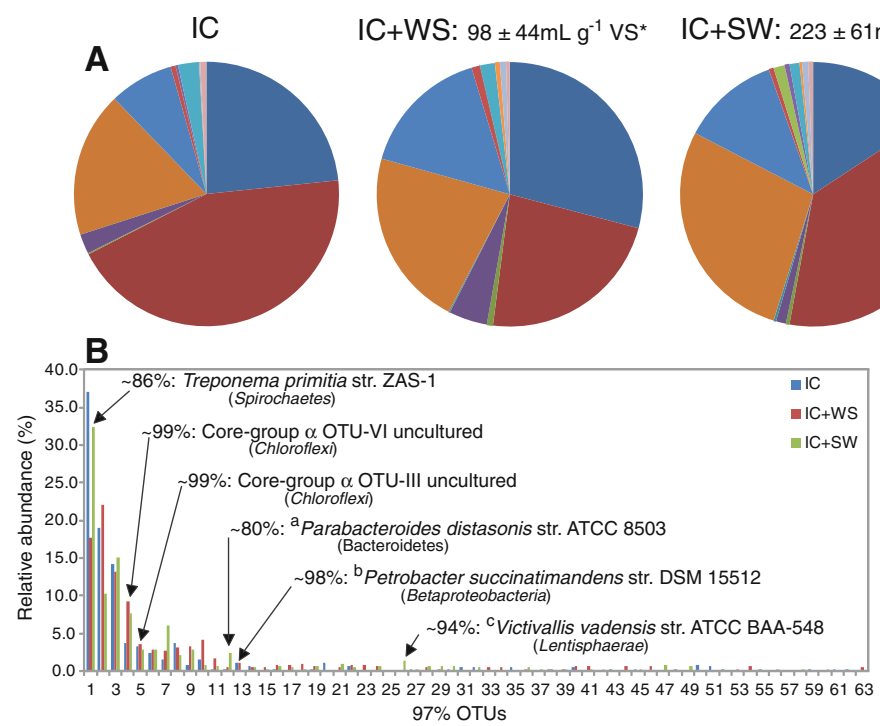

Fig. 3 Relative abundance and comparison profiles of bacterial 16S rRNA OTUs identified in anaerobic digesters containing either waste water sludge with no additional organic substrate (inoculum, IC), IC plus wheat straw (IC $+\mathrm{WS}$ ), or IC plus seaweed $(\mathrm{IC}+\mathrm{SW})$. Relative abundance of bacterial lineages at a phylum-level (a) and OTU-level (b) are shown. OTU abundance shifts between WS and SW digesters (c) were measured as either fold-change increases (+) or decreases (-) against IC measurements. Lineage information for selected OTUs (a-c) and OTU affiliation to previously described,

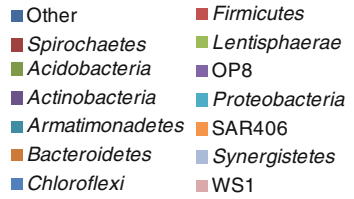

c

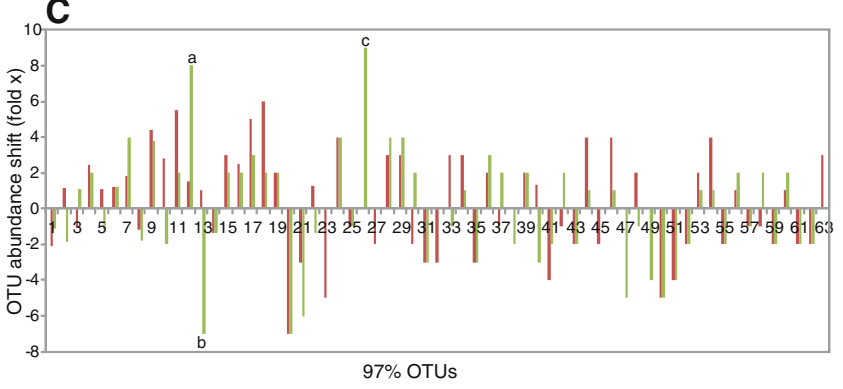

highly prevalent core phylotypes (Rivière et al. 2009) is provided. Colour coding in $\mathbf{b}$ and $\mathbf{c}$ are as follows: IC, red indicates IC + WS and green indicates IC $+\mathrm{SW}$. OTUs numbers in the $x$-axis corresponds to BAC_nor-terminology referred to in the text. Total methane yields are included in a for IC $+\mathrm{WS}$ and $\mathrm{IC}+\mathrm{SW}$, which are provided in the original publication on methane production (Vivekanand et al. 2012) and normalized for production in IC. $V S^{*}$ volatile solids

\section{References}

ARC_nor-1 dominance is conceivably linked to bacterial population shifts and/or changes in bacterial metabolism. Surprisingly, dominant bacterial populations showed little variation between the digesters with larger shifts only observed for several low-abundant OTUs. Regardless, the large phylogenetic variation between biogas-producing communities and cultured representatives makes drawing definitive functional or interactive conclusions, a significant challenge. The functioning of biogas-producing microbial communities on the whole is insufficiently explored and requires further in depth structure-function analysis involving a combination of cultivation directed strategies and "meta-omic" approaches (i.e., metagenomics, metatranscriptomics).

Acknowledgments This work was supported by The Research Council of Norway's RENERGI program (Grant numbers 190877) and a Marie Curie International Incoming Fellowship from the European Commission (awarded to PBP; PIIF-GA-2010-274303).

Conflict of interest The authors declare that they have no conflict of interest.

Open Access This article is distributed under the terms of the Creative Commons Attribution License which permits any use, distribution, and reproduction in any medium, provided the original author(s) and the source are credited.
Caporaso JG, Kuczynski J, Stombaugh J, Bittinger K, Bushman FD, Costello EK, Fierer N (2010) QIIME allows integration and analysis of high-throughput community sequencing data. Nat Meth 7:335-336

Cole JR, Chai B, Marsh TL, Farris RJ, Wang Q, Kulam SA, Chandra S, McGarrell DM, Schmidt TM, Garrity GM, Tiedje JM (2003) The Ribosomal Database Project (RDP-II): previewing a new autoaligner that allows regular updates and the new prokaryotic taxonomy. Nucleic Acids Res 31:442-443

Edgar RC (2010) Search and clustering orders of magnitude faster than BLAST. Bioinformatics 26:2460-2461

Edgar RC, Haas BJ, Clemente JC, Quince C, Knight R (2011) UCHIME improves sensitivity and speed of chimera detection. Bioinformatics 27:2194-2200

Ellis JT, Tramp C, Sims RC, Miller CD (2012) Characterization of a methanogenic community within an algal fed anaerobic digester. ISRN Microbiol. doi:10.5402/2012/753892

Gantner S, Andersson AF, Alonso-Sáez L, Bertilsson S (2011) Novel primers for $16 \mathrm{~S}$ rRNA-based archaeal community analyses in environmental samples. J Microbiol Methods 84:12-18

Hamady M, Walker JJ, Harris JK, Gold NJ, Knight R (2008) Errorcorrecting barcoded primers for pyrosequencing hundreds of samples in multiplex. Nat Meth 5:235-237

Horn SJ (2009) Seaweed biofuels: production of biogas and bioethanol from brown macroalgae. VDM Verlag. ISBN 978-3-639-15307-1

Horn SJ, Ostgaard K (2001) Alginate lyase activity and acidogenesis during fermentation of Laminaria hyperborea. J Appl Phycol 13:143-152 
Kampmann K, Ratering S, Kramer I, Schmidt M, Zerr W, Schnell S (2012) Unexpected stability of bacteroidetes and firmicutes communities in laboratory biogas reactors fed with different defined substrates. Appl Environ Microbiol 78:2106-2119

Nelson MC, Morrison M, Yu Z (2011) A meta-analysis of the microbial diversity observed in anaerobic digesters. Bioresour Technol 102:3730-3739

Nielsen HB, Heiske S (2011) Anaerobic digestion of macroalgae: methane potentials, pre-treatment, inhibition and co-digestion. Water Sci Technol 64:1723-1729

Pope PB, Mackenzie AK, Gregor I, Smith W, Sundset MA, McHardy AC, Morrison M, Eijsink VGH (2012) Metagenomics of the svalbard reindeer rumen microbiome reveals abundance of polysaccharide utilization loci. PLoS One 7:e38571

Rivière D, Desvignes V, Pelletier E, Chaussonnerie S, Guermazi S, Weissenbach J, Li T, Camacho P, Sghir A (2009) Towards the definition of a core of microorganisms involved in anaerobic digestion of sludge. ISME J 3:700-714

Rosewarne CP, Pope PB, Denman SE, McSweeney CS, O'Cuiv P, Morrison M (2010) High-yield and phylogenetically robust methods of DNA recovery for metagenomic analysis of the microbial biofilms adherent to plant biomass in the herbivore gut. Microb Ecol 61:448-454

Vivekanand V, Eijsink VGH, Horn SJ (2012) Biogas production from the brown seaweed Saccharina latissima: thermal pretreatment and codigestion with wheat straw. J Appl Phycol 24:1295-1301

Zoetendal EG, Plugge CM, Akkermans AD, de Vos WM (2003) Victivallis vadensis gen. nov., sp. nov., a sugar-fermenting anaerobe from human faeces. Int $J$ Syst Evol Microbiol $53: 211-215$ 